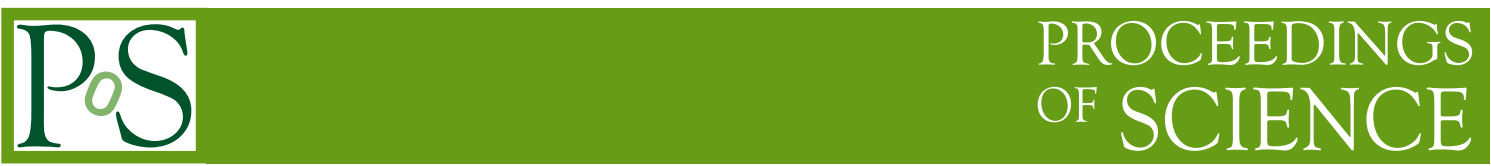

\title{
phi2 and phi3 measurements at Belle
}

\author{
J. Dalseno*ab \\ ${ }^{a}$ Max-Planck-Institut für Physik \\ Föringer Ring 6 \\ München 80805 Germany \\ ${ }^{b}$ Technische Universität München \\ Excellence Cluster Universe \\ Boltzmannstraße 2 \\ Garching 85748 Germany \\ E-mail: jdalseno@mpp.mpg.de
}

We present a summary of the measurements of the CKM angles $\phi_{2}$ and $\phi_{3}$, performed by the Belle experiment which collects $B \bar{B}$ pairs at the $\Upsilon(4 S)$ resonance produced in asymmetric-energy $e^{+} e^{-}$collisions. We discuss the most precise measurements of the ADS observables $\mathscr{R}_{D K}$ and $\mathscr{A}_{D K}$ as well as the first model-independent determination of $\phi_{3}$ in the GGSZ method. We also speculate on the precision that can be achieved with the final Belle data set on the $\phi_{2}$ related channels $B^{0} \rightarrow \pi^{+} \pi^{-}, \rho^{0} \rho^{0}$, and $a_{1}^{ \pm} \pi^{\mp}$.

The 2011 Europhysics Conference on High Energy Physics-HEP 2011,

July 21-27, 2011

Grenoble, Rhône-Alpes France

*Speaker. 


\section{Introduction}

The main goal of the Belle experiment at KEK is to constrain the unitarity triangle for $B$ decays. This allows us to test the Cabibbo-Kobayashi-Maskawa (CKM) mechanism for violation of the combined charge-parity $(C P)$ symmetry $[1,2]$, as well as search for new physics effects beyond the Standard Model (SM). These proceedings give a summary of the experimental status of measurements of the CKM phases $\phi_{2}$ and $\phi_{3}$, defined from CKM matrix elements as $\phi_{2} \equiv$ $\arg \left(-V_{t d} V_{t b}^{*}\right) /\left(V_{u d} V_{u b}^{*}\right)$ and $\phi_{3} \equiv \arg \left(-V_{u d} V_{u b}^{*}\right) /\left(V_{c d} V_{c b}^{*}\right)$.

First-order weak processes (tree) proceeding by $b \rightarrow u \bar{u} d$ quark transitions such as $B^{0} \rightarrow \pi \pi$, $\rho \pi, \rho \rho$ and $a_{1}^{ \pm} \pi$, are directly sensitive to $\phi_{2}$. In the quasi-two-body approach, CKM angles can be determined by measuring the time-dependent asymmetry between $B^{0}$ and $\bar{B}^{0}$ decays [3]. For the decay sequence $\Upsilon(4 S) \rightarrow B_{C P} B_{\mathrm{Tag}} \rightarrow f_{C P} f_{\mathrm{Tag}}$, where one of the $B$ mesons decays at time $t_{C P}$, to a $C P$ eigenstate $f_{C P}$, and the other decays at time $t_{\text {Tag }}$, to a flavour specific final state $f_{\text {Tag }}$, with $q=+1(-1)$ for $B_{\mathrm{Tag}}=B^{0}\left(\bar{B}^{0}\right)$, the decay rate has a time-dependence given by

$$
P(\Delta t, q)=\frac{e^{-|\Delta t| / \tau_{B^{0}}}}{4 \tau_{B^{0}}}\left[1+q\left(\mathscr{A}_{C P} \cos \Delta m_{d} \Delta t+\mathscr{S}_{C P} \sin \Delta m_{d} \Delta t\right)\right],
$$

where $\Delta t \equiv t_{C P}-t_{\mathrm{Tag}}$ and $\Delta m_{d}$ is the mass difference between the $B_{H}$ and $B_{L}$ mass eigenstates. The parameters, $\mathscr{A}_{C P}$ and $\mathscr{S}_{C P}$, describe direct and mixing-induced $C P$ violation, respectively.

If a single first-order weak amplitude dominates the decay, then we expect $\mathscr{A}_{C P}=0$ and $\mathscr{S}_{C P}=$ $\sin 2 \phi_{2}$. On the other hand, if second-order loop processes (penguins) are present, then direct $C P$ violation is possible, $\mathscr{A}_{C P} \neq 0$. Additionally, as these loop processes are not directly proportional to $V_{u b}$, our measurement of $\mathscr{S}_{C P}$ does not directly determine $\phi_{2}$, rather $\mathscr{S}_{C P}=\sqrt{1-\mathscr{A}_{C P}^{2}} \sin \left(2 \phi_{2}-\right.$ $\left.2 \Delta \phi_{2}\right)$, where $\Delta \phi_{2}$ is the shift caused by the second order contributions.

A theoretically clean way of accessing $\phi_{3}$ is through $B^{-} \rightarrow D K^{-}$decays where $D$ represents an admixture of $D^{0}$ and $\bar{D}^{0}$ states. This is possible through an interference if the $D$ decays to a common final state $|D\rangle=\left|D^{0}\right\rangle+r_{B} e^{i \theta}\left|\bar{D}^{0}\right\rangle$, where $\theta \equiv \delta_{B} \pm \phi_{3}$ is the relative phase difference between the two processes for $B^{+}$and $B^{-}$in which $\delta_{B}$ is the relative strong phase difference in $B$ decays. The quantity $r_{B}$, is the amplitude ratio $A\left(B^{-} \rightarrow \bar{D}^{0} K^{-}\right) / A\left(B^{-} \rightarrow D^{0} K^{-}\right)$, and should be around the order of colour suppression as the two processes are of similar strength in the Cabibbo angle $\lambda$.

\section{2. $B^{-} \rightarrow D K^{-}, D \rightarrow K^{+} \pi^{-}$}

In the so-called ADS method [4], $B^{-} \rightarrow D K^{-}$with $D \rightarrow K^{+} \pi^{-}$and the charge conjugate decays are used. Here, the favoured $B$ decay $(b \rightarrow c)$ followed by the doubly CKM-suppressed $D$ decay interferes with the suppressed $B$ decay $(b \rightarrow u)$ followed by the CKM-favoured $D$ decay. The relative similarity of the combined decay amplitudes enhances the possible $C P$ asymmetry.

The ADS variables are defined as,

$$
\begin{aligned}
\mathscr{R}_{D K} & \equiv \frac{\mathscr{B}\left(\left[K^{+} \pi^{-}\right] K^{-}\right)+\mathscr{B}\left(\left[K^{-} \pi^{+}\right] K^{+}\right)}{\mathscr{B}\left(\left[K^{-} \pi^{+}\right] K^{-}\right)+\mathscr{B}\left(\left[K^{+} \pi^{-}\right] K^{+}\right)}, \\
\mathscr{A}_{D K} & \equiv \frac{\mathscr{B}\left(\left[K^{+} \pi^{-}\right] K^{-}\right)-\mathscr{B}\left(\left[K^{-} \pi^{+}\right] K^{+}\right)}{\mathscr{B}\left(\left[K^{+} \pi^{-}\right] K^{-}\right)+\mathscr{B}\left(\left[K^{-} \pi^{+}\right] K^{+}\right)},
\end{aligned}
$$



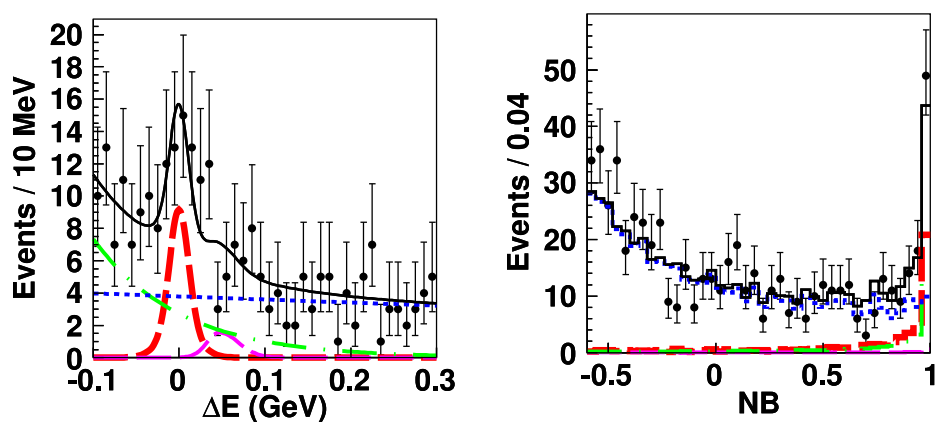

Figure 1: $\Delta E(N B>0.9)$ and $N B(|\Delta E|<0.03 \mathrm{GeV})$ distributions for the suppressed $B^{-} \rightarrow D_{\text {Sup }}\left[K^{+} \pi^{-}\right] K^{-}$. The red curve shows the signal, the magenta curve the $D \pi$ component, the green curve the $B \bar{B}$ background and the blue curve the continuum.

which are related to $\phi_{3}$ as

$$
\begin{aligned}
\mathscr{R}_{D K} & =r_{B}^{2}+r_{D}^{2}+2 r_{B} r_{D} \cos \left(\delta_{B}+\delta_{D}\right) \cos \phi_{3}, \\
\mathscr{A}_{D K} & =\frac{2 r_{B} r_{D} \sin \left(\delta_{B}+\delta_{D}\right) \sin \phi_{3}}{\mathscr{R}_{D K}},
\end{aligned}
$$

where the amplitude ratio $r_{D}=A\left(D^{0} \rightarrow K^{+} \pi^{-}\right) / A\left(\bar{D}^{0} \rightarrow K^{+} \pi^{-}\right)$, and $\delta_{D}$ is the strong phase difference between the two $D$ amplitudes.

This analysis has been performed with the final Belle data set containing 772 million $B \bar{B}$ pairs. The main difficulty is in separating the small signal from the dominant continuum background and is achieved by a fit to the kinematic variable $\Delta E$, and a neural network output $N B$, based on the event shape. First evidence for the suppressed $B^{-} \rightarrow D_{\text {Sup }}\left[K^{+} \pi^{-}\right] K^{-}$was found at a $4.1 \sigma$ significance as shown in Fig. 1. From this measurement, the ADS observables were found to be

$$
\begin{aligned}
\mathscr{R}_{D K} & =\left[1.63_{-0.41}^{+0.44}(\text { stat })_{-0.13}^{+0.07}(\text { syst })\right] \times 10^{-2}, \\
\mathscr{A}_{D K} & \left.=-0.39_{-0.28}^{+0.26}(\text { stat })_{-0.03}^{+0.04} \text { (syst }\right) .
\end{aligned}
$$

which was the most precise measurement at the time of publication [5].

$$
\text { 3. } B^{-} \rightarrow D K^{-}, D \rightarrow K_{S}^{0} \pi^{+} \pi^{-}
$$

In the so-called GGSZ method [6], $B^{-} \rightarrow D^{(*)} K^{-}$decays where the $D$ decays to the $C P$ eigenstate $D \rightarrow K_{S}^{0} \pi^{+} \pi^{-}$, are used. One can fit the $D \rightarrow K_{S}^{0} \pi^{+} \pi^{-}$Dalitz plot with the matrix element $\left|\mathscr{M}_{ \pm}\left(m_{+}^{2}, m_{-}^{2}\right)\right|^{2}=\left|f_{D}\left(m_{+}^{2}, m_{-}^{2}\right)+r_{B} e^{i\left(\delta_{B} \pm \phi_{3}\right)} f_{D}\left(m_{-}^{2}, m_{+}^{2}\right)\right|^{2}$, thereby determining $\phi_{3}$ directly in the fit. The amplitude $f_{D}$, which depends on the invariant squared masses $m_{ \pm}\left(K_{S}^{0} \pi^{ \pm}\right)$is typically parametrised as the coherent sum of 2-body decays via intermediate resonances and also measured in the fit.

This measurement has been performed previously at Belle using 657 million $B \bar{B}$ pairs [7]. By combining the results of $B^{-} \rightarrow D K^{-}$and $D^{*} K^{-}$, where $D^{*} \rightarrow D \pi^{0}$ and $D \gamma, \phi_{3}=\left(78_{-12}^{+11}\right.$ (stat) \pm 4 (syst) $\pm 9($ model $))^{\circ}$ was obtained. Note that the dominant systematic uncertainty arises from model dependence in the parametrisation of $f_{D}$ which would eventually dominate the total uncertainty at $\mathrm{LHCb}$ and the next generation $B$ factories. 

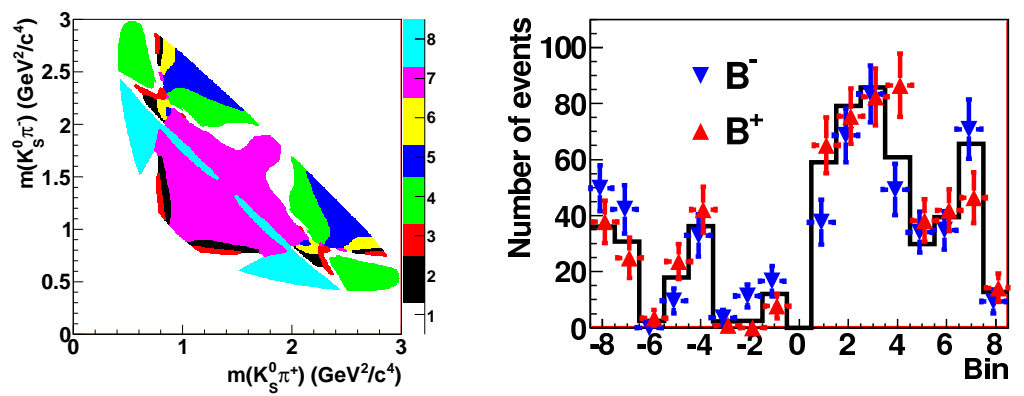

Figure 2: The left figure shows the optimised binning where the colours represent different bins. The right figure shows the fitted $B^{-} \rightarrow D K^{-}$yields determined in each Dalitz plot bin as the data points, while the solid curve shows the expected yield in each bin.

A new method removing the model uncertainty has recently been developed [8] which involves binning the Dalitz plot and working with the measured number of signal events in each bin instead. This can be compared in a $\chi^{2}$ fit with the expected number of events in each bin $i$,

$$
N_{i}^{ \pm}=h_{B}\left[K_{i}+r_{B}^{2} K_{-i}+2 \sqrt{K_{i} K_{-i}}\left(x_{ \pm} c_{i}+y_{ \pm} s_{i}\right)\right],
$$

where $x_{ \pm}=r_{B} \cos \left(\delta_{B} \pm \phi_{3}\right)$ and $y_{ \pm}=r_{B} \sin \left(\delta_{B} \pm \phi_{3}\right)$ are free parameters in the fit, constraining the phase $\phi_{3}$, and $h_{B}$ is a normalisation constant. Here, $K_{i}$ is the number of events in bin $i$ determined from a flavour-tagged sample $D^{* \pm} \rightarrow D \pi^{ \pm}$, while $c_{i}=\left\langle\cos \Delta \delta_{D}\right\rangle_{i}$ and $s_{i}=\left\langle\sin \Delta \delta_{D}\right\rangle_{i}$ are related to average strong phase difference in bin $i$ and are measured by CLEO [9], but can also be measured at BES-III in the future.

Compared to measuring $\left|f_{D}\right|^{2}$, a binned analysis reduces the statistical precision of $\phi_{3}$, but this can be optimised. The advantage of this method is that the optimal binning depends on the model, however $\phi_{3}$ does not. Studies show that the precision depends strongly on the amplitude behaviour across the bins. Better precision can be achieved when the phase difference between the $D^{0}$ and $\bar{D}^{0}$ amplitudes varies as little as possible. The optimised binning was found using the amplitude measured by BaBar [10] and is shown in Fig. 2.

This analysis has been performed with the final Belle data set of 772 million $B \bar{B}$ pairs which obtained a total signal yield of $1176 \pm 43$ events. Following this, the signal yield in the optimised Dalitz plot bins is determined then compared in a $\chi^{2}$ fit with the expected signal yield given in Eq. 3.1. A significant $C P$ asymmetry can be seen in Fig. 2 which has a $0.4 \%$ probability of being a statistical fluctuation.

The parameters $x_{ \pm}$and $y_{ \pm}$are determined in the fit, constraining $\phi_{3}, r_{B}$ and $\delta_{B}$,

$$
\begin{aligned}
& \phi_{3}=\left(77.3_{-14.9}^{+15.1} \pm 4.2 \pm 4.3\right)^{\circ}, \\
& r_{B}=0.145 \pm 0.030 \pm 0.011 \pm 0.011, \\
& \delta_{B}=(129.9 \pm 15.0 \pm 3.9 \pm 4.7)^{\circ},
\end{aligned}
$$

where the first error is statistical, the second systematic and the third is the precision on $c_{i}$ and $s_{i}$ from CLEO. This is a promising proof of concept as the precision on $\phi_{3}$ is comparable to the previous measurement with $B^{-} \rightarrow D K^{-}$only. 
4. $B^{0} \rightarrow \pi^{+} \pi^{-}, \rho^{0} \rho^{0}, a_{1}^{ \pm} \pi^{\mp}$

These analyses are ongoing at Belle and will be based on the final data set. Improvements have been made to the tracking algorithm and fitting methods to improve the detection efficiency. We expect our final results for $B^{0} \rightarrow \pi^{+} \pi^{-}$and $a_{1}^{ \pm} \pi^{\mp}$ to be the most precise measurements when they are released and at least an improved upper limit is expected on $B^{0} \rightarrow \rho^{0} \rho^{0}$ which will have an impact on $\phi_{2}$.

\section{References}

[1] N. Cabibbo, Phys. Rev. Lett. 10, 531 (1963).

[2] M. Kobayashi and T. Maskawa, Prog. Theor. Phys. 49, 652 (1973).

[3] I. Bigi and A. Sanda, CP Violation, Cambridge University Press, Cambridge (2009).

[4] D. Atwood, I. Dunietz, and A. Soni, Phys. Rev. Lett. 78, 3257 (1997).

[5] Y. Horii et al. [ Belle Collaboration ], Phys. Rev. Lett. 106, 231803 (2011).

[6] A. Giri, Yu. Grossman, A. Soffer, J. Zupan, Phys. Rev. D 68, 054018 (2003); A. Bondar, Proceedings of BINP Special Analysis Meeting on Dalitz Analysis, 24-26 Sep. 2002, unpublished.

[7] A. Poluektov et al. [ Belle Collaboration ], Phys. Rev. D 81, 112002 (2010).

[8] A. Bondar and A. Poluektov, Eur. Phys. J. C 47, 347 (2006); Eur. Phys. J. C 55, 51 (2008).

[9] J. Libby et al. [ CLEO Collaboration ], Phys. Rev. D 82, 112006 (2010).

[10] B. Aubert, et al. [ BaBar Collaboration ], Phys. Rev. D 78, 034023 (2008). 